\title{
Numerical simulation of pulsed liquid fluidized bed and its experimental validation
}

\author{
Guodong Jin*, Yongsheng Nie, Dayou Liu \\ Institute of Mechanics, Chinese Academy of Sciences, Institute of Mechanics 15 Zhongguancun Road Beijing 100080, People's Republic of China
}

Received 4 May 2000; received in revised form 6 November 2000

\begin{abstract}
The pulsed liquid fluidized bed was studied using numerical simulation and experimental methods. The area-averaged two-fluid model (TFM) was used to simulate the pulsed fluidization. The bed expansion and collapse processes were simulated first and the phenomena obtained from the calculation were consistent with our previous experiments and observations. In the pulsed fluidization, the variation of bed height, the variations of particle velocity and concentration distribution were obtained and analyzed. Experiments were carried out to validate the simulation results. The pressure variation with time at different locations was measured using pressure transducers and compared with the simulated results. The variations of bed height and particle concentration distribution were recorded using a digital video camera recorder. The results were consistent with the simulation results as a whole. C) 2001 Elsevier Science B.V. All rights reserved.
\end{abstract}

Keywords: Pulsed fluidized beds; Two-fluid model; Numerical simulation; Experiment

\section{Introduction}

For many years, the main factor influencing the performance of fluidized beds has been the low gas-solid contact efficiency caused by the existing of bubbles. Various means have been employed to reduce the effect of bubbles, such as special distributors, insertion of baffles and packing. Lots of studies show that fluidization quality can be much improved by externally applied vibrations or pulses [1-4]. There have been many experimental studies of pulsed fluidized beds, but the theoretical understanding of pulsed fluidized beds is far from complete. Quantitative understanding the pulsed fluidization is needed for its industry application.

We have carried out the study of pulsed fluidized beds in recent years and our main interest is dynamics and physical mechanisms. In our early research works, for simplicity, we divided the whole process of pulsed fluidization into an expansion process and a collapsing process [5]. We have intensively studied the expansion and collapsing processes. Two mathematical models have been

\footnotetext{
* Corresponding author.
}

proposed to simulate expansion and collapsing processes, respectively. The models were also used to calculate the pressure variation in pulsed gas-solid fluidized beds with low frequency. The simulation results were in good agreement with the experimental data [6]. When the pulse frequency is high, the bed starts to collapse while it does not expand completely and starts to expand while it does not collapse completely. The previous models are not suitable for this situation.

Our final goal is to use a general model that can be used to simulate pulsed fluidized beds with any frequency. But it is very difficult to simulate bubbles behavior in the bed. So we began our study from the simulation of liquid fluidized beds. In this paper, we will use the area-averaged one-dimensional TFM to simulate the whole process of pulsed fluidization of liquid-solid beds with any frequency.

The two-fluid model (TFM) was introduced by many authors [7-9] to describe two-phase systems considering the particle and fluid phases as interpenetrating continua. The main problem of the two-fluid model is that most of the constitutive relations, such as the relations between stress and strain of solid phase and the interaction between the solid and fluid phases, are difficult to establish [10] and some assumptions must be introduced into the model. Tsuo 
and Gidaspow [11] computed the flow pattern in circulating fluidized beds (CFB) using the two-fluid model. Gera et al. [12] simulated bubbles in large-particle fluidized bed with the two-fluid model and the distinct element model (DEM). Although the DEM has some advantages in simulating the interactions among particles, if one simulates a system with millions of particle, the DEM will take much more time as compared to the TFM. The DEM is not possible to simulate a fluidized bed of full industrial scale with the limitation of the computer. Di Felice [13] used the TFM to predict the solid, liquid and gas three-phase system; they treated the liquid and gas phases as a single homogeneous fluid. However, up to now, no literature about the simulation and quantitative analysis of pulsed fluidization using the TFM has been found.

\section{Mathematical model}

The model is based on the following assumptions:

1. The flow is one-dimensional.

2. Fluid is incompressible.

3. All the particles have the same size.

4. The irregular motion and collision of particles are ignored.

5. The friction on the wall is neglected, for it is far less than the gravity of particles.

The continuity and momentum equations for solid and fluid phases are, respectively [14],

$\frac{\partial \alpha_{\mathrm{p}}}{\partial t}+\frac{\partial\left(\alpha_{\mathrm{p}} u_{\mathrm{p}}\right)}{\partial x}=0$,

$\frac{\partial \alpha_{\mathrm{f}}}{\partial t}+\frac{\partial\left(\alpha_{\mathrm{f}} u_{\mathrm{f}}\right)}{\partial x}=0$.

$\rho_{\mathrm{p}}\left(\frac{\partial\left(\alpha_{\mathrm{p}} u_{\mathrm{p}}\right)}{\partial t}+\frac{\partial\left(\alpha_{\mathrm{p}} u_{\mathrm{p}}^{2}\right)}{\partial x}\right)=-\alpha_{\mathrm{p}} \frac{\partial p}{\partial x}-\alpha_{\mathrm{p}} \rho_{\mathrm{p}} g+F_{\mathrm{p}}$,

$\rho_{\mathrm{f}}\left(\frac{\partial\left(\alpha_{\mathrm{f}} u_{\mathrm{f}}\right)}{\partial t}+\frac{\partial\left(\alpha_{\mathrm{f}} u_{\mathrm{f}}^{2}\right)}{\partial x}\right)=-\alpha_{\mathrm{f}} \frac{\partial p}{\partial x}-\alpha_{\mathrm{f}} \rho_{\mathrm{f}} g-F_{\mathrm{p}}$,

where $\alpha_{\mathrm{p}}$ and $\alpha_{\mathrm{f}}$ are the volume fraction of solid and fluid phases, respectively, $\alpha_{\mathrm{p}}+\alpha_{\mathrm{f}}=1 . F_{\mathrm{p}}$ is the interaction force per unit volume between the solid and fluid phases; it can be expressed [15],

$F_{\mathrm{p}}=\left(\rho_{\mathrm{p}}-\rho_{\mathrm{f}}\right) g \alpha_{\mathrm{p}}\left(1-\alpha_{\mathrm{p}}\right)^{(1-n)}\left(u_{\mathrm{f}}-u_{\mathrm{p}}\right) / u_{T}$,

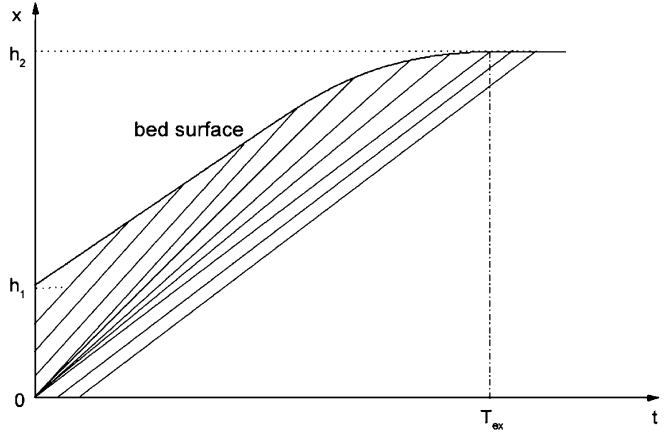

Fig. 1. Map of wave series in expansion process.

where the exponent $n$ depends on the particle Reynolds number, $u_{T}$ is particle setting velocity in a stable liquid and defined as,

$u_{T}=\left(\frac{\rho_{\mathrm{p}}-\rho_{\mathrm{f}}}{\rho_{\mathrm{p}}}\right) g \tau$,

and the relaxation time of a particle $\tau$ is defined as,

$\tau=\frac{d_{\mathrm{p}}^{2} \rho_{\mathrm{p}}}{k \mu_{\mathrm{f}}}$.

Adding Eq. (1) to Eq. (2) and integrating it along the bed height yields,

$\alpha_{\mathrm{p}} u_{\mathrm{p}}+\left(1-\alpha_{\mathrm{p}}\right) u_{\mathrm{f}}=U(t)$,

where $U(t)$ is the instantaneous liquid flux and is a given function.

Using Eqs. (3) and (4), an equation without pressure term can be obtained,

$$
\begin{aligned}
& \alpha_{\mathrm{f}} \rho_{\mathrm{p}}\left(\frac{\partial\left(\alpha_{\mathrm{p}} u_{\mathrm{p}}\right)}{\partial t}+\frac{\partial\left(\alpha_{\mathrm{p}} u_{\mathrm{p}}^{2}\right)}{\partial x}\right) \\
& -\alpha_{\mathrm{p}} \rho_{\mathrm{f}}\left(\frac{\partial\left(\alpha_{\mathrm{f}} u_{\mathrm{f}}\right)}{\partial t}+\frac{\partial\left(\alpha_{\mathrm{f}} u_{\mathrm{f}}^{2}\right)}{\partial x}\right) \\
& =\left(\rho_{\mathrm{f}}-\rho_{\mathrm{p}}\right) \alpha_{\mathrm{f}} \alpha_{\mathrm{p}} g+F_{\mathrm{p}} .
\end{aligned}
$$

Adding Eq. (3) to Eq. (4), one can get the pressure equation,

$$
\begin{aligned}
-\frac{\partial p}{\partial x}= & \rho_{\mathrm{p}}\left(\frac{\partial\left(\alpha_{\mathrm{p}} u_{\mathrm{p}}\right)}{\partial t}+\frac{\partial\left(\alpha_{\mathrm{p}} u_{\mathrm{p}}^{2}\right)}{\partial x}\right) \\
& +\rho_{\mathrm{f}}\left(\frac{\partial\left(\alpha_{\mathrm{f}} u_{\mathrm{f}}\right)}{\partial t}+\frac{\partial\left(\alpha_{\mathrm{f}} u_{\mathrm{f}}^{2}\right)}{\partial x}\right) \\
& +\left(\rho_{\mathrm{f}} \alpha_{\mathrm{f}}+\rho_{\mathrm{p}} \alpha_{\mathrm{p}}\right) g .
\end{aligned}
$$




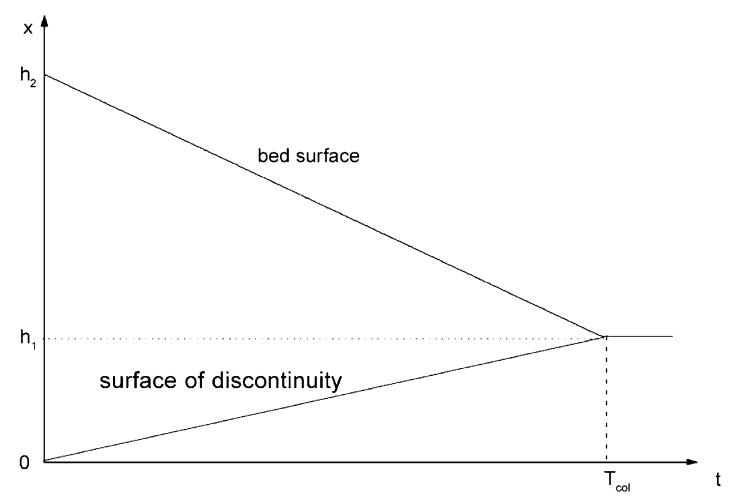

Fig. 2. Surface of discontinuity in collapse process.

The boundary conditions are:

$x=0, u_{\mathrm{p}}(t, 0)=0, \quad \alpha_{\mathrm{p}}(t, 0)=1-\left(\frac{U(t)}{u_{T}}\right)^{1 /(n+1)} ;$

$x=h(t), u_{\mathrm{p}}(t, h)=\frac{\mathrm{d} h}{\mathrm{~d} t} ;$

$x=H_{\text {bed }}, p\left(t, H_{\text {bed }}\right)=0$.

The initial conditions are:

$t=0: \quad \alpha_{\mathrm{p}}(0, x)=\alpha_{\mathrm{p}}^{0}, \quad u_{\mathrm{p}}(0, x)=0$.

Particle concentration $\alpha_{\mathrm{p}}(t, x)$, velocity $u_{\mathrm{p}}(t, x)$ and liquid velocity $u_{\mathrm{f}}(t, x)$ can be obtained by solving the set of Eqs. (1), (8), (9), (11), (12) and (14); pressure $p$ can be calculated by using Eqs. (10) and (13).

The finite difference method was used to solve the set of equations. A BTBS (backward time and backward space) difference scheme was used. The Euler coordinate

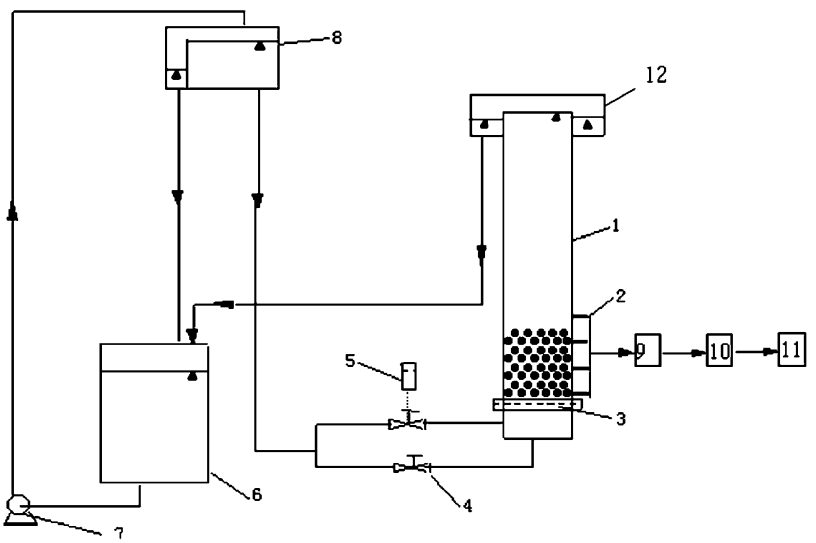

Fig. 3. Schematic diagram of the experimental apparatus. (1) Fluidized bed; (2) pressure transducer; (3) plate distributor; (4) spheric valve; (5) time-lag relay and electromagnetic valve; (6) lower water tank; (7) centrifugal pump; (8) upper water tank; (9) amplifier; (10) A/D converter; (11) computer; (12) water gathering vessel.
Table 1

Physical properties of the particles used

\begin{tabular}{lllll}
\hline Particles & $d_{\mathrm{p}}(\mathrm{mm})$ & $\rho_{\mathrm{p}}\left(\mathrm{kg} / \mathrm{m}^{3}\right)$ & $u_{T}(\mathrm{~m} / \mathrm{s})$ & $n$ \\
\hline Glass beans & 1.8 & 2500 & 0.172 & 1.37 \\
\hline
\end{tabular}

system was used except the node on the upper boundary $(x=h(t))$. The upper boundary of the calculation region, i.e., the interface between the single liquid phase region and the particle-liquid two-phase region is a moving boundary during a pulsed fluidization process. The position of the upper boundary is determined using Eq. (12), the concentration and velocity on the upper boundary is calculated using a special scheme (see the Appendix).

\section{Brief introduction to Slis model}

Slis et al. [16] have studied the simple expansion and collapse processes using analysis method. The equations they used included Eqs. (1) and (8) and the following equation, ${ }^{1}$

$U-u_{\mathrm{p}}=u_{T} \alpha_{\mathrm{f}}^{(n+1)}$.

It is a generalization of the relationship of Richardson and Zaki [17], i.e., $U=u_{T} \alpha_{\mathrm{f}}^{(n+1)}$ for a steady fluidization.

Using the Eqs. (1), (8) and (15), they have demonstrated that there is a concentration wave propagating with velocity $V$ in the fluidized bed,

$V=\left[(n+1) \alpha_{\mathrm{f}}^{n}-(n+2) \alpha_{\mathrm{f}}^{n+1}\right] u_{T}+U$.

During the propagation of the wave, the concentration on the wavefront is constant.

$\frac{\partial \alpha_{\mathrm{f}}}{\partial t}+V \frac{\partial \alpha_{\mathrm{f}}}{\partial x}=0$.

The map of the wave series on the $x-t$ plane (Fig. 1) can be given using Eqs. (15)-(17) to study the expansion process, with the flux $U$ varying from $U=U_{1}$ to $U_{2}>U_{1}$. The expansion duration is,

$T_{\mathrm{ex}}=\frac{h_{2} \alpha_{\mathrm{f}, 2}}{U_{2}(n+1)\left(1-\alpha_{\mathrm{f}, 2}\right)}$,

where $h_{2}$ is the terminate height of the bed after it expanses completely, and $\alpha_{\mathrm{f}, 2}$ is the fluid fraction corresponding to $U=U_{2}$.

If the sudden expansion disturbance is replaced with a series of small expansion disturbances, from Fig. 1 and Eq. (16), one can know that the propagation velocity of a later

\footnotetext{
${ }^{1}$ The exponent $n$ in literature [16] equals $n+1$ in this paper.
} 


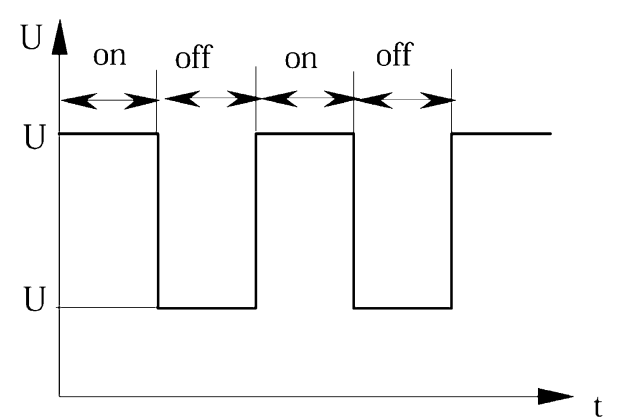

Fig. 4. Illustration diagram of flow rate varying with time.

small expansion disturbance is slower than that of the earlier one. The variation of all variables in the bed is continual.

When we study the collapse process, with the flux $U$ changing from $U=U_{2}$ to $U=U_{1}<U_{2}$, if the sudden collapse disturbance is replaced with a series of small collapse disturbances, from Eq. (16), one can know that the propagation velocities of later small collapse disturbances are quicker than those of the earlier ones. The later disturbances overlie the earlier ones in the propagation process, and a certain limited intensive discontinuity is formed. Each variable has a discontinuity on both sides of the surface of discontinuity (Fig. 2). The collapse duration that Slis et al. [16] got using analysis method is,

$T_{\mathrm{col}}=\frac{h_{2}\left(\alpha_{\mathrm{f}, 2}-\alpha_{\mathrm{f}, 1}\right)}{\left(1-\alpha_{\mathrm{f}, 1}\right)\left(U_{2}-U_{1}\right)}=\frac{h_{2}-h_{1}}{U_{2}-U_{1}}$,

where $h_{1}$ is the terminate height of the bed after it collapses completely, and $\alpha_{\mathrm{f}, 1}$ is the fluid fraction corresponding to $U=U_{1}$. The propagation velocity of the discontinuity surface is,

$V_{\mathrm{s}}=\frac{\left(U_{1}-U_{2}\right)\left(1-\alpha_{\mathrm{f}, 1}\right)}{\left(\alpha_{\mathrm{f}, 1}-\alpha_{\mathrm{f}, 2}\right)}$.

Slis et al. [16] have studied the single expansion and collapse processes using this model in uniform solid-liquid fluidized bed, and the results were well consistent with their experimental results.

In the pulsed fluidization, the expansion and collapse processes alternatively take place and there is a complex interaction among the concentration wave series. The Slis model has not been used to simulate the pulsed fluidization up to now.

Comparing the Slis model with the equation set in Section 2, the two models are very similar. Neglecting the inertia terms on the left hand of Eq. (9) and introducing Eq. (5) of the interaction force between the two phases, one can get the same equation as Eq. (15).

One can know that the Slis model is the simplification of the TFM neglecting the inertia terms. The pressure distribution can be calculated using Eq. (10). Because the inertia was neglected, there is a discontinuity between the upper section, $\alpha_{\mathrm{f}}=\alpha_{\mathrm{f}, 2}, u_{\mathrm{p}}=U_{1}-U_{2}$, and the lower section, $\alpha_{\mathrm{f}}=\alpha_{\mathrm{f}, 1}, u_{\mathrm{p}}=0$, in the collapse process. When the inertia is considered from above to below, there is a transition section from $\alpha_{\mathrm{f}}=\alpha_{\mathrm{f}, 2}$, to $\alpha_{\mathrm{f}}=\alpha_{\mathrm{f}, 1}$, and the order of the transition section is $\tau|W|$, where $W$ is the particle velocity above the surface of discontinuity. In the expansion process, there is a very thick transition section between the upper and lower sections even when using the Slis model. The thickness of this transition section is much larger than $\tau|W|$. The characters of the transition section in expansion are hardly changed neglecting the inertia.

\section{Experimental apparatus}

Fig. 3 is the schematic diagram of the experimental apparatus. The experiments were carried out under ambient conditions in a 29-mm ID and 1150-mm-high Perspex column fitted with a porous plate distributor. The pressures at different locations of the bed were measured simultaneously by four pressure transducers. The signals were collected and treated by a computer after magnification and A/D conversion, and the sampling frequency was $100 \mathrm{~Hz}$. (a)

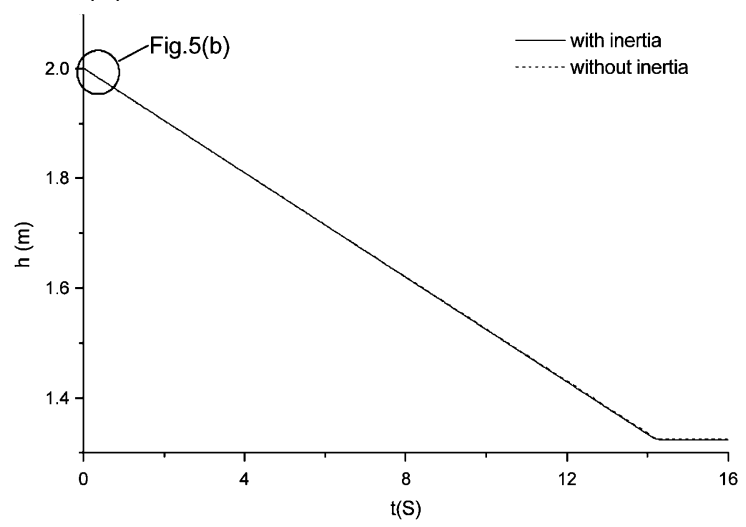

(b)

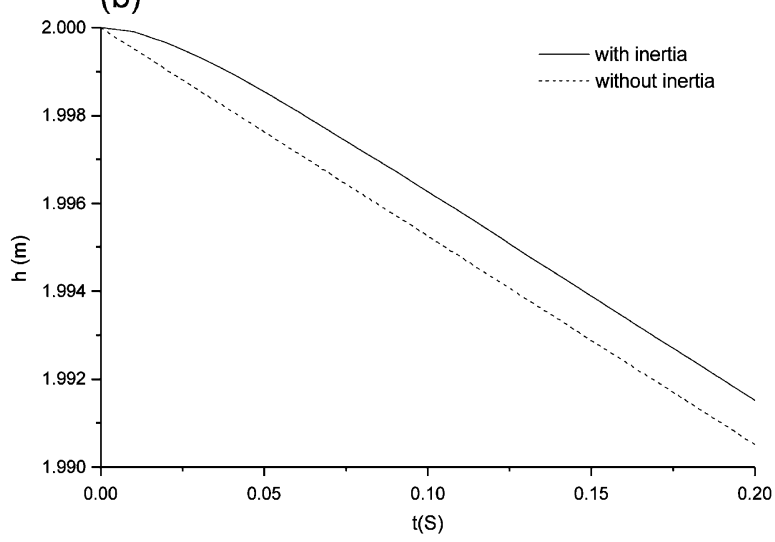

Fig. 5. Bed height variation with time in collapse process. 


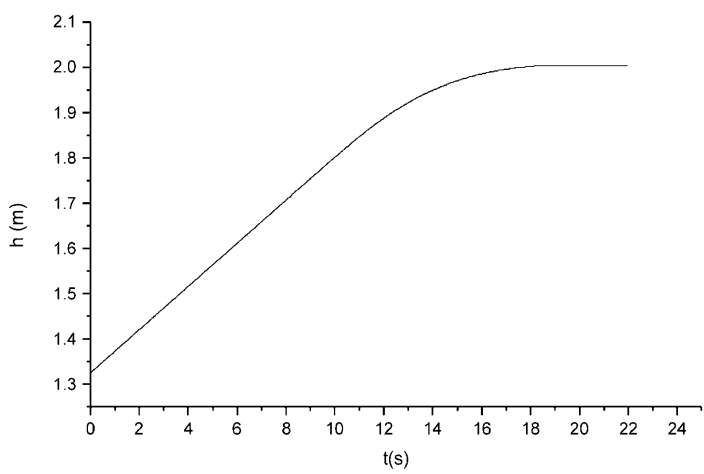

Fig. 6. Bed height variation with time in expansion process.

The variations of bed height and particle concentration were recorded using a digital video camera recorder. The fluid used was water. The properties of particles used are shown in Table 1.

Particles were fluidized by pulsed liquid. The liquid flowing into the bed includes two parts: one was steady flow and the other was pulsed flow. The flux was measured using a turbine flowmeter. The ideal variation of flux with time is illustrated in Fig. 4. The pulse frequency, the time of on-period and off-period were controlled by the graded time-lag relay.

\section{Results and discussion}

\subsection{Simulation of collapsing process and expansion pro- cess}

In order to verify the model proposed in this paper, we simulated the bed collapsing process resulting from a sudden decrease of the fluid flux from $U_{2}$ to $U_{1}$, and the bed expansion process resulting from a sudden increase of fluid flux from $U_{1}$ to $U_{2}$.

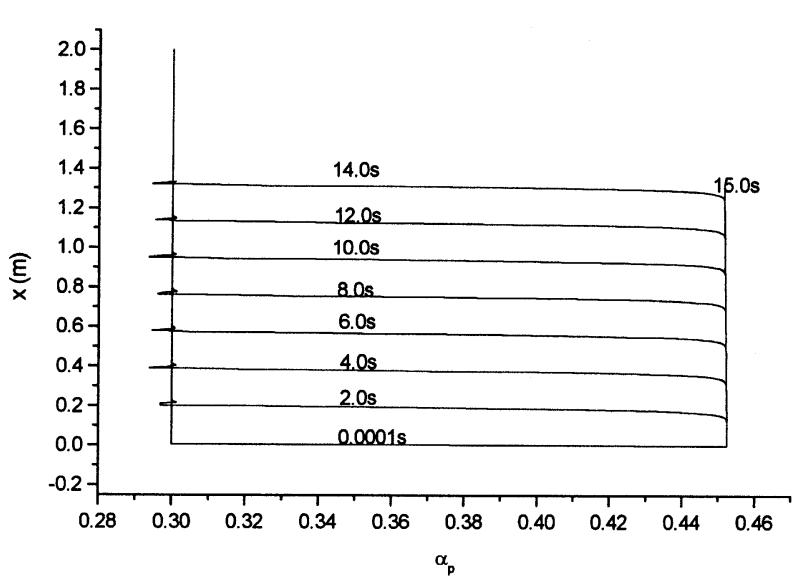

Fig. 7. Distribution of particle concentration in collapse process.

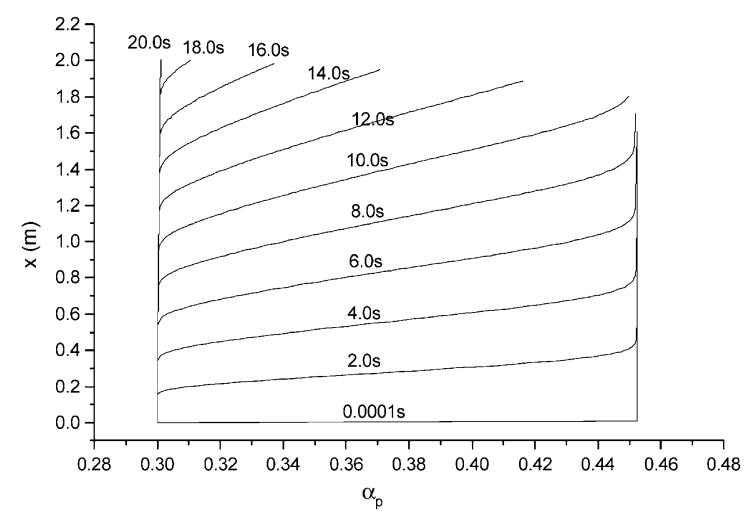

Fig. 8. Distribution of particle concentration in expansion process.

Figs. 5 and 6 are the simulated variation of the bed surface $h(t)$ with time in collapsing process and expansion process, respectively. The shape of $h-t$ curve for collapsing process is nearly a straight line (Fig. 5(a)), but at the beginning of the line there is short transformation period because of the inertia (the solid line in Fig. 5(b)). Fig. 5(b) shows the difference between the result gotten from our model and that from the Slis model [16]. The inertia takes effects only at the incipient period $(\delta t \approx \tau)$; as a whole, the two results are so similar that the two lines almost parallel each other. The line gotten from our model is delayed by a period of $\tau$ as compared to the line gotten from Slis model (Fig. 5(b)).

Fig. 7 is the variation and distribution of particle concentration in different times during the collapsing process, where $x$ is the space coordinate measured vertically upward from the bottom of the bed. For example, at $t=4 \mathrm{~s}$, from above to below, particle concentration $\alpha_{\mathrm{p}}(t, x)$ sharply transits from $\alpha_{\mathrm{p}, 2}$, which accords to the initial particle concentration before the collapse to $\alpha_{\mathrm{p}, 1}$, which accords to the ultimate particle concentration after the collapse, the thickness of the transition section is very thin. The velocity of the transition section (or the surface of

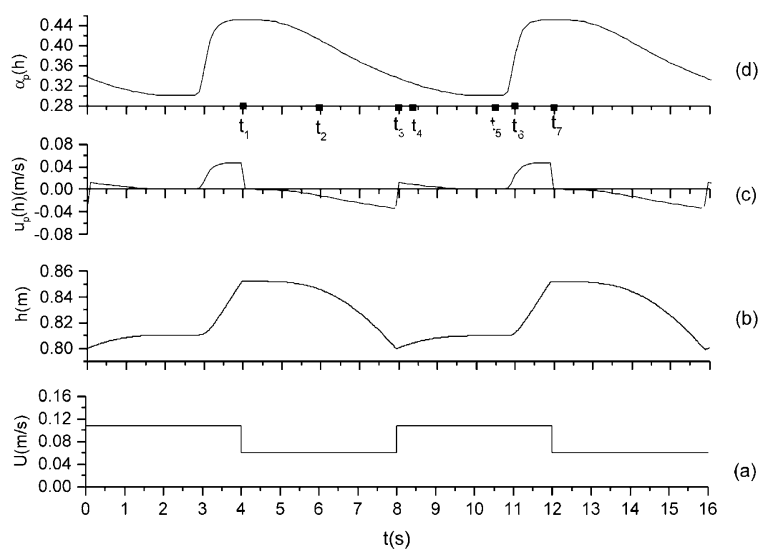

Fig. 9. Variation of characteristic variables at the bed surface with time, in case A (on-period $4 \mathrm{~s}$, off-period $4 \mathrm{~s}$ ). 


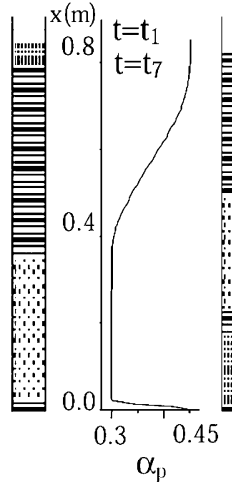

(a)

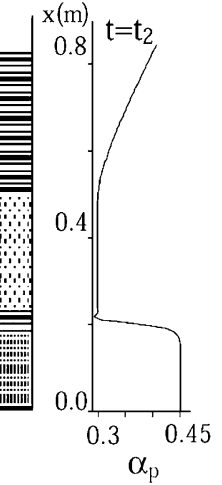

(b)

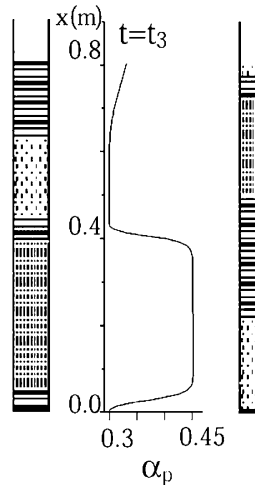

(c)

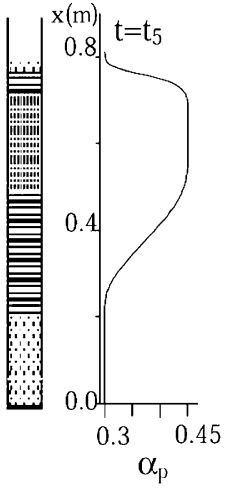

(d)

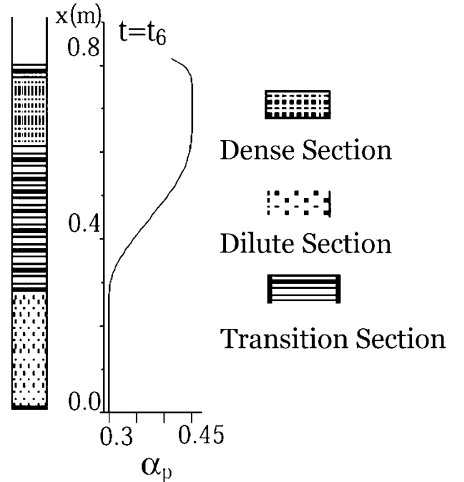

(e)

Fig. 10. Distribution of particle concentration in the pulsed fluidized bed in case A.

discontinuity) is consistent with Eq. (20); the bed surface falls at the same time. When the surface of discontinuity reaches the bed surface, the particle concentration of the whole bed becomes $\alpha_{\mathrm{p}, 1}$, and the collapsing process ends.

Fig. 8 is the variation and distribution of particle concentration during expansion process at different times. For example, at $t=4 \mathrm{~s}$, from above to below, particle concentration $\alpha_{\mathrm{p}}(t, x)$ gradually transits from $\alpha_{\mathrm{p}, 1}$, which accords to the initial particle concentration before the expansion to $\alpha_{\mathrm{p}, 2}$, which accords to the ultimate particle concentration after the expansion, the thickness of the transition section is much thicker. The transition section broadens on its way up the bed; the bed surface travels upward at the same time. From Fig. 6, one can see that the bed height $h(t)$ increases with time $t$ linearly at the beginning and the curve begins to blend when the upper of transition section reaches the bed surface. When the lower of the transition section travels through the bed surface, the particle concentration of the whole bed uniformly equals $\alpha_{\mathrm{p}, 2}$; the expansion process ends.

Comparing Figs. 5 and 7 with Figs. 6 and 8, one can see the essential differences between the collapsing process and the expansion process.

The simulated results are consistent with Slis et al. [16] and our previous experimental observation [5].

\subsection{Simulation of the pulsed fluidized beds}

When the fluid flux changes periodically and the onperiod and the off-period are less than the expansion duration and the collapse duration, respectively, the bed starts to collapse while it does not expanse completely and starts to expanse while it does not collapse completely. Thus, the pulsed fluidization occurs.

We simulated the pulsed fluidized beds with different on-periods and off-periods. The variations of the bed surface $h(t)$, particle concentration distribution $\alpha_{\mathrm{p}}(t, x)$ and pressure distribution $p(t, x)$ were obtained from the calculations.

In case $\mathrm{A}$, the on-period is not long enough for the bed to expand completely and the off-period is not long enough for the bed to collapse completely. The variations of liquid flux, bed height, particle velocity and particle concentration at bed surface with time are shown in Fig. 9(a),(b),(c) and (d). The distribution of particle concentration at different times is shown in Fig. 10. In this example, the on-period for expansion and the off-period for collapse are both $4 \mathrm{~s}$, while it takes about $9 \mathrm{~s}$ to expand completely and about $7 \mathrm{~s}$ to collapse completely.

At $t=t_{1}$, the distribution of particle concentration in the bed is shown in Fig. 10(a). When the flux decreases suddenly from $U_{2}$ to $U_{1}$ during a transitory period from $t_{1-}$ to $t_{1+}$, a very thin transition section that can be regarded as a discontinuity appears near the distributor. The distribution of particle concentration in the bed almost remains the same, $\alpha_{\mathrm{p}}\left(t_{1+}, x\right)=\alpha_{\mathrm{p}}\left(t_{1-}, x\right)$. From the Eq. (15), we know that $u_{\mathrm{p}}\left(t_{1+}, x\right)-u_{\mathrm{p}}\left(t_{1-}, x\right)=U\left(t_{1+}\right)-$ $U\left(t_{1-}\right)=U_{1}-U_{2}$. But it changes from $\alpha_{\mathrm{p}}=\alpha_{\mathrm{p}, 2}$ to $\alpha_{\mathrm{p}}=$ $\alpha_{\mathrm{p}, 1}$, from the lower side of the discontinuity surface to the upper side near the distributor. The variation of particle velocity at the bed surface, $u_{\mathrm{p}}\left(t_{+}, h\left(t_{+}\right)\right)-u_{\mathrm{p}}\left(t_{-}, h\left(t_{-}\right)\right)$, also equals $U_{1}-U_{2}$ (see Fig. 9(c)).

From time $t=t_{1}$ to $t=t_{2}$, the discontinuity surface travels to the position $x=0.20 \mathrm{~m} . \alpha_{\mathrm{p}}=\alpha_{\mathrm{p}, 1}$ and $u_{\mathrm{p}}=0$

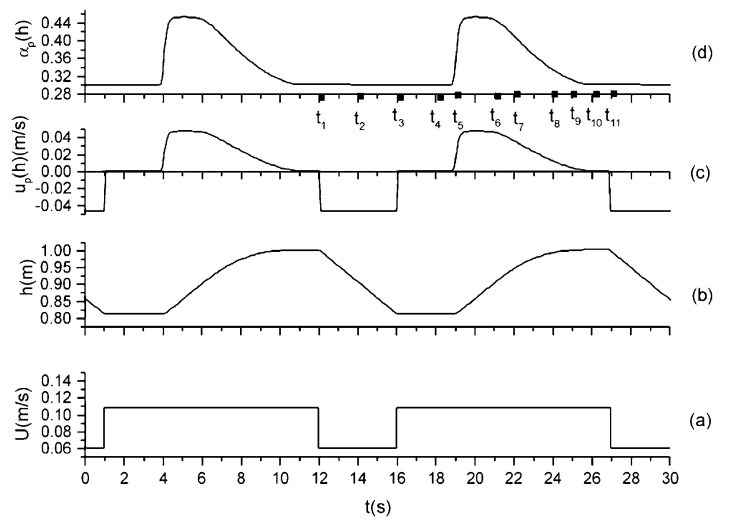

Fig. 11. Variation of characteristic variables at the bed surface with time, in case B (on-period $11 \mathrm{~s}$, off-period $4 \mathrm{~s}$ ). 


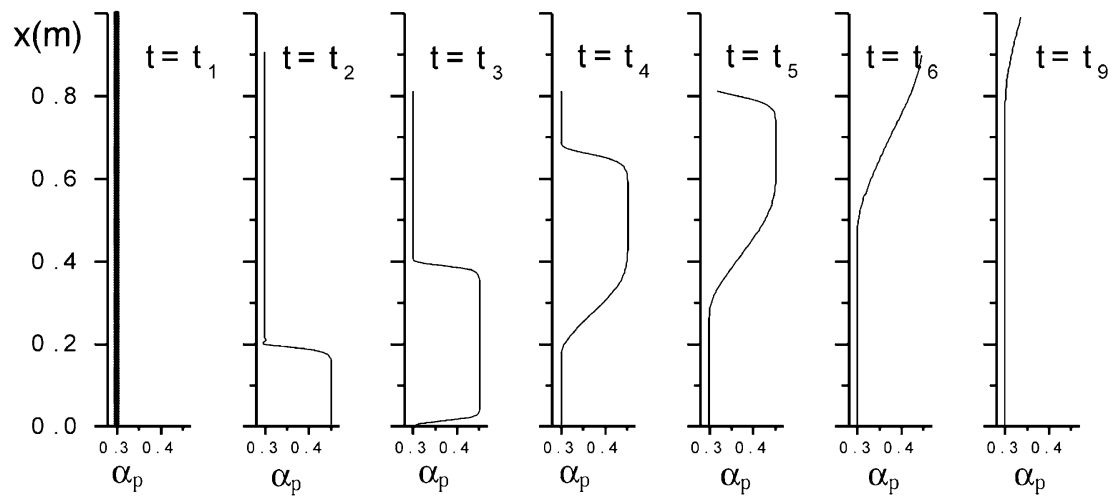

Fig. 12. Distribution of particle concentration in the pulsed fluidized bed in case B.

in the section between the discontinuity surface and the distributor. The dilute section above the discontinuity surface and the transition section produced in the expansion process travels upward accordingly. The bed surface $(x=$ $h(t))$ that lies in the upper part of the transition section a moment ago is in the middle of it (Fig. 10(b)). The velocity of the bed surface decreases with the decreasing of the particle concentration at the bed surface (Eq. (15); Fig. 9(c) and (d)), and the falling velocity becomes faster gradually (Eq. (15) and Fig. 9(c)).

From time $t=t_{2}$ to $t=t_{3}$, the discontinuity surface, the dilute section and the transition section continue to travel upward, and the bed surface is in the lower part of the transition section. $\alpha_{\mathrm{p}}(t, h(t))$ and $u_{\mathrm{p}}(t, h(t))$ also continue to decrease and the falling velocity of the bed surface becomes much larger.

During the transitory period from $t=t_{3-}$ to $t=t_{3+}$, the flux increases suddenly from $U_{1}$ to $U_{2}$, the particle concentration near the distributor decreases from $\alpha_{\mathrm{p}, 1}$ to $\alpha_{\mathrm{p}, 2}$ accordingly, while the most section of particle concentration in the bed keeps the same, but the particle velocity $u_{\mathrm{p}}(t, x)$ increases suddenly by $\left(U_{2}-U_{1}\right)$ (Eq. (15) and Fig. 9(c)). With the sign of $u_{\mathrm{p}}(t, h)$ changing from negative to positive, a turning point appears in the curve of the bed surface (Fig. 9(b)), and the whole bed begins to travel upward with the velocity in equilibrium to local particle concentration (Eq. (15)).

From time $t=t_{3}$ to $t=t_{5}$, a transition section is formed gradually near the distributor and travels upward, the bed surface is out of the transition section and goes into the dilute section. At time $t=t_{5}$, there is a dilute section above the distributor (Fig. 10(d)) and the discontinuity surface reaches the bed surface.

From time $t=t_{5}$ to $t=t_{6}$, the discontinuity surface travels across the bed surface, the particle concentration at the bed surface changes from $\alpha_{\mathrm{p}, 1}$ to $\alpha_{\mathrm{p}, 2}$ quickly, and a turning point occurs in the curve of the bed surface line (Fig. 9(b)). At time $t=t_{7}$, the flow state comes back to that at time $t=t_{1}$, a cycle ends, and another begins.

In case $\mathrm{B}$, the on-period is long enough for the bed to expand completely but the off-period is not long enough for the bed to collapse completely. The results are shown in Figs. 11 and 12.

At time $t=t_{1}$, the whole bed has a uniform particle concentration corresponding to $U_{2}$ and the particle velocity equals zero. Then the liquid flux decreases suddenly from

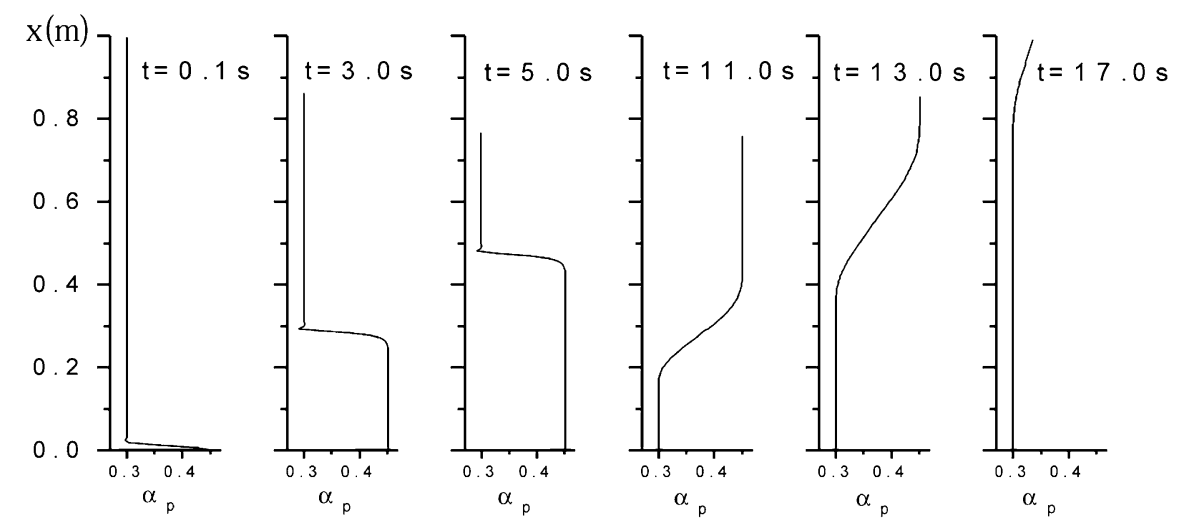

Fig. 13. Distribution of particle concentration in the pulsed fluidized bed in case C. 


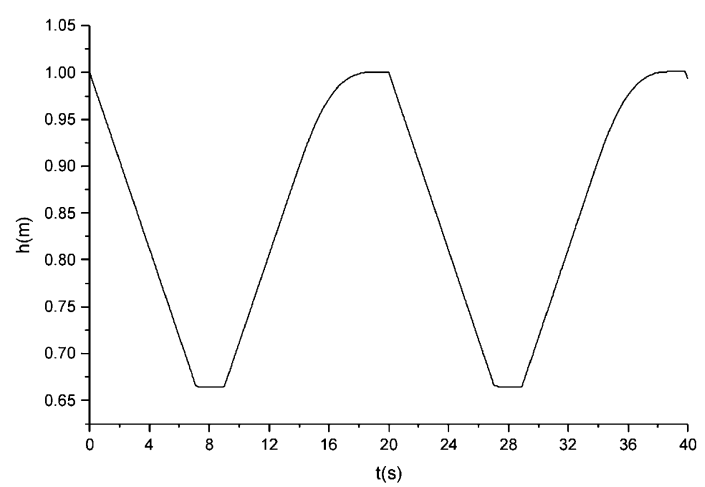

Fig. 14. Bed height variation with time in case C (on-period $11 \mathrm{~s}$, off-period $9 \mathrm{~s}$ ).

$U_{2}$ to $U_{1}$ and the particles begin to move downwards. A section with higher particle concentration appears near the bottom and becomes thicker and thicker. But the particle concentration and velocity keep constant above the section (Fig. 12).

At time $t=t_{3}$, the flux increases suddenly from $U_{1}$ to $U_{2}$ but the particle concentration along the bed cannot change suddenly; however, the particle velocity throughout the bed increases suddenly by $\left(U_{2}-U_{1}\right)$. The dense section with particle concentration $\alpha_{\mathrm{p}, 1}$ begins to travel upward with the speed of $u_{\mathrm{p}}=\left(U_{2}-U_{1}\right)$, and the velocity of the dilute section with particle concentration $\alpha_{\mathrm{p}, 2}$ becomes zero again. The bed height does not change from time $t=t_{3}$ until the dense section reaches the bed surface at time $t=t_{5}$ (Fig. 11(b)). At the time $t=t_{5+}$, a sudden change happens to the velocity of the bed surface, from $u_{\mathrm{p}}(t, h)=0$ to $u_{\mathrm{p}}(t, h)=U_{2}-U_{1}$.

From time $t=t_{6}$ on, the bed surface goes into the transition section after the dense section travels out of the bed surface, and the particle concentration at the bed surface decreases gradually with the velocity decreasing accordingly. At time $t=t_{10}$, the transition section travels through the bed surface and the expansion process completes; the whole bed is fluidized uniformly with

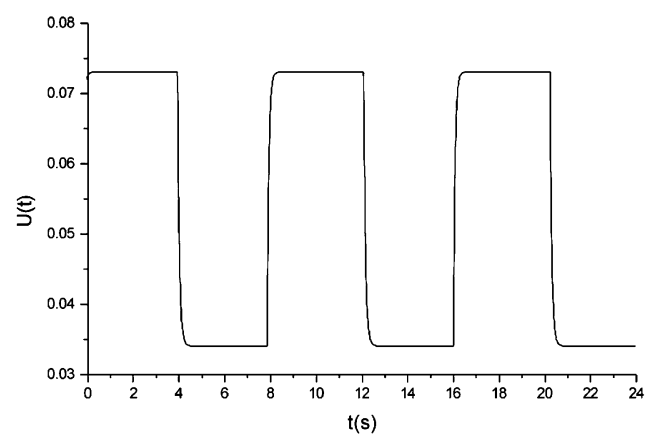

Fig. 15. The flux variation in calculation to comparing with experiments.

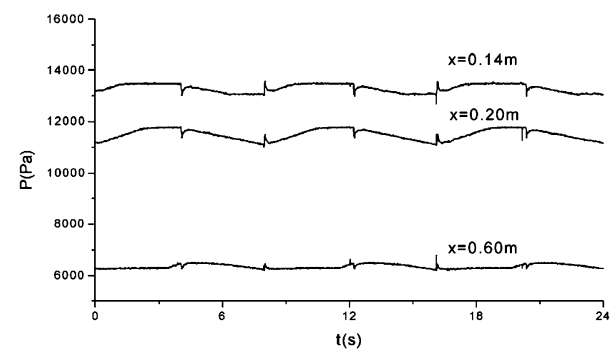

Fig. 16. Measured pressure variation with time at different locations.

$u_{\mathrm{p}}(t, x)=0$. This state remains constant until $t=t_{11}$; another cycle begins.

In case $\mathrm{C}$, both the on-period and off-period are long enough for the bed to expand and collapse completely. The variations of the particle concentration distribution $\alpha_{\mathrm{p}}(t$, $x$ ) and the bed height $h(t)$ with time along the bed, are shown in Figs. 13 and 14, respectively. It is a combination of expansion and collapsing processes. The flow behavior is similar to the above cases.

\subsection{Comparing simulated results with experimental results}

In order to validate the simulated results, the pulsed fluidization experiments were carried out. The flux variation is shown in Fig. 15. The variation of bed height $h(t)$ and the distribution of particle concentration $\alpha_{\mathrm{p}}(t, x)$ were recorded using a digital video camera recorder. The pressure variations with time at some positions along the fluidized bed, $p\left(t, x_{i}\right)(i=1,2,3,4)$, were measured using pressure transducers (Fig. 16). The comparisons of variation of bed height $h(t)$, particle concentration distribution $\alpha_{\mathrm{p}}(t, x)$, and pressures $p\left(t, x_{i}\right)$ are shown in Figs. 17-19. From Figs. 17-19, one can know that the calculation results provide a good agreement with the experiment results. From Fig. 16, one can know that a pressure pulse will appear when the flux changes suddenly. This is the contribution of the inertia term at the right hand of Eq. (9). The amplitude of the pulse is in inverse ratio to the characteristic time of the flux changing process. The pres-

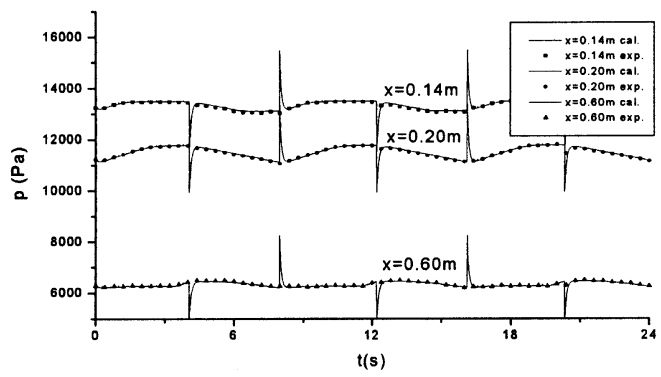

Fig. 17. Comparison of pressure between the measured and simulated results at different locations. 

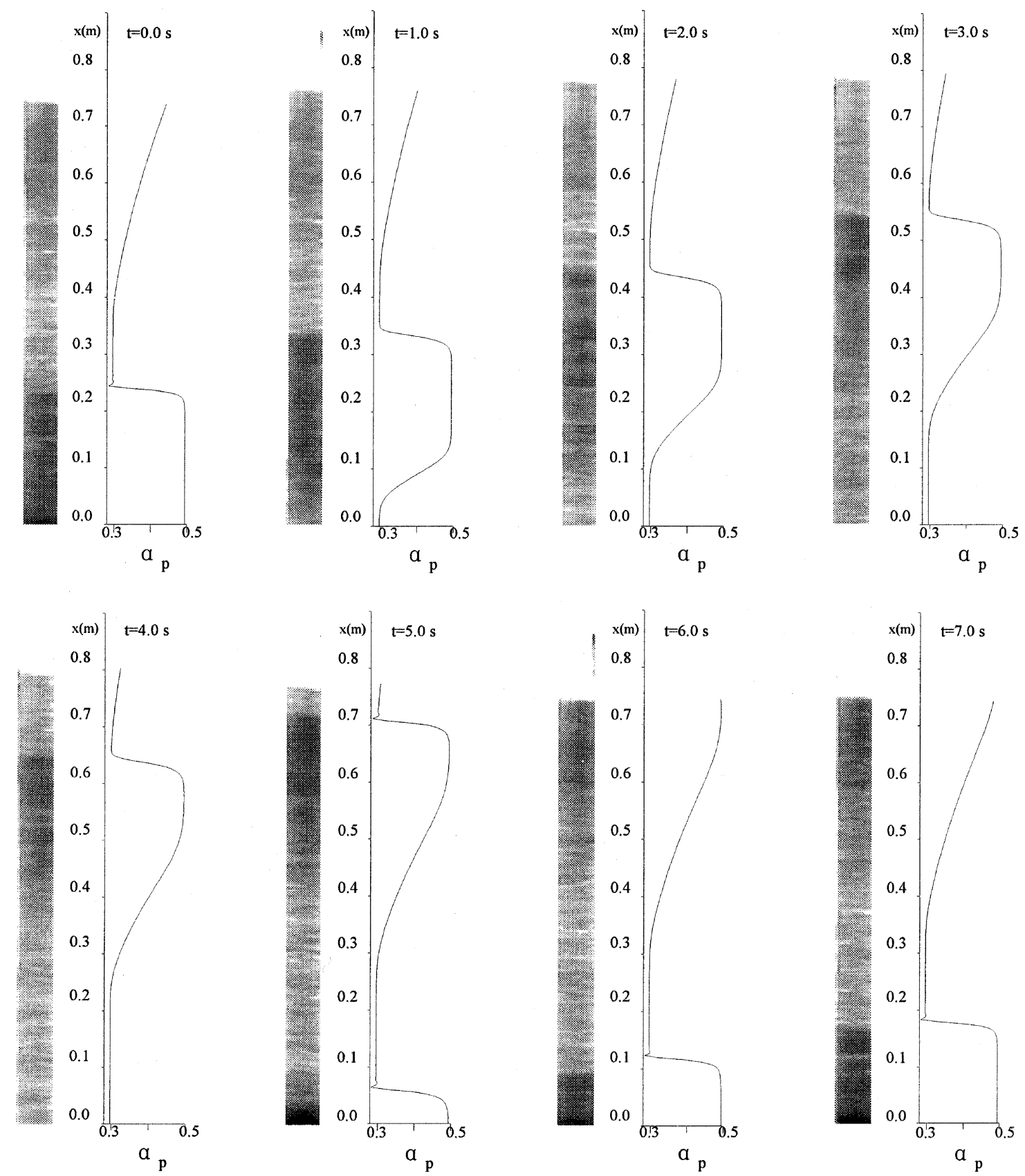

Fig. 18. The comparison of particle concentration distribution between the experimental and simulated results at different time.

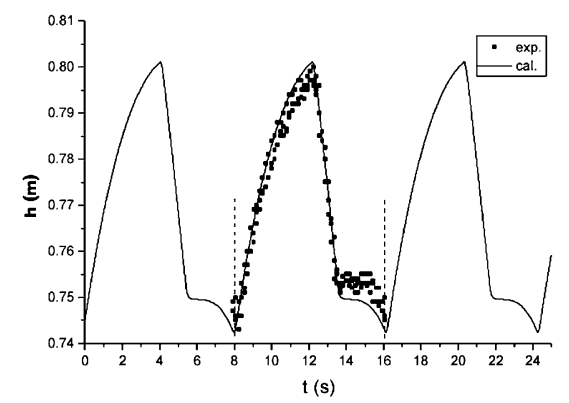

Fig. 19. Comparison of bed height between the simulated and experimental results. sure variation in a certain position is caused by the total amount of the particles above it, in addition to the inertia effect.

\section{Conclusion}

(1) The simulation results of the two-fluid model suggested in this paper are in good agreement with the experiment results. It is shown that the model can well describe the variation processes in the pulsed fluidized bed 
and the assumptions introduced in this paper are reasonable.

(2) This model is also suitable to the gas-solid pulsed fluidized bed, if there are no bubbles or the bubbles are too small to influence the flow uniformity in the bed.

(3) A dense section and a dilute section appear in the pulsed fluidized bed alternately. The particle concentration at a certain position alters between the dense and the dilute concentrations, which may be the reason that pulse fluidized bed can effectively prevent the origination of the gas bubbles. Even though there are some gas bubbles in the pulse fluidized bed, it is difficult for them to penetrate through the dense section, which may be the mechanism that the pulsed fluidized bed can prevent the gas bubbles from growing larger.

\section{Notation}

\section{English letters}

$d_{\mathrm{p}} \quad$ particle diameter $(\mathrm{m})$

$F_{\mathrm{p}} \quad$ the interaction between the particle and the liquid phases per unit volume $\left(\mathrm{N} \mathrm{m}^{-3}\right)$

$g$ gravitational acceleration $\left(\mathrm{m} \mathrm{s}^{-2}\right)$

$H_{\text {bed }} \quad$ the highest point of the bed column (m)

$h \quad$ height of the fluidized bed $(\mathrm{m})$

$k \quad$ constant in Eq. (7)

$n \quad$ constant in Eq. (5)

$p$ pressure $(\mathrm{Pa})$

$P_{\text {bed }} \quad$ pressure at the highest point of bed column $(\mathrm{Pa})$

$t \quad$ time coordinate (s)
$U_{1} \quad$ liquid flux in off-period $\left(\mathrm{m} \mathrm{s}^{-1}\right)$

$U_{2} \quad$ liquid flux in on-period $\left(\mathrm{m} \mathrm{s}^{-1}\right)$

$U$ instantaneous liquid flux $\left(\mathrm{m} \mathrm{s}^{-1}\right)$

$u_{\mathrm{f}} \quad$ liquid velocity $\left(\mathrm{m} \mathrm{s}^{-1}\right)$

$u_{\mathrm{p}} \quad$ particle velocity $\left(\mathrm{m} \mathrm{s}^{-1}\right)$

$u_{\mathrm{t}} \quad$ particle setting velocity in stable liquid $\left(\mathrm{m} \mathrm{s}^{-1}\right)$

$x \quad$ space coordinate $(\mathrm{m})$

\section{Greek letters}

$\alpha_{\mathrm{f}} \quad$ liquid fraction

$\alpha_{\mathrm{p}} \quad$ particle fraction

$\alpha_{\mathrm{p}}^{0} \quad$ initial particle fraction

$\alpha_{\mathrm{p}, 1} \quad$ particle fraction in stable fluidized state corresponding to the flux $U_{1}$

$\alpha_{\mathrm{p}, 2} \quad$ particle fraction in stable fluidized state corresponding to the flux $U_{2}$

$\mu_{\mathrm{f}} \quad$ viscosity coefficient (Pa s)

$\rho_{\mathrm{p}} \quad$ particle density $\left(\mathrm{kg} \mathrm{m}^{-3}\right)$

$\rho_{\mathrm{f}} \quad$ liquid density $\left(\mathrm{kg} \mathrm{m}^{-3}\right)$

$\tau \quad$ particle relaxation time (s)

\section{Acknowledgements}

We are grateful for the support from the Multiphase Reaction Laboratory, Institute of Chemical Metallurgy, Chinese Academy of Sciences and the State Key Laboratory of Multiphase Flow in Power Engineering, Xi'an Jiaotong University. We are also grateful for the help from Guozheng Li, who helped to improve our sampling system.

\section{Appendix A}

The difference scheme of Eqs. (1) and (9) for inner nodes are,

$$
\begin{aligned}
\alpha_{\mathrm{p}, i}^{n+1}= & \alpha_{\mathrm{p}, i}^{n}-\frac{\alpha_{\mathrm{p}, i}^{n} u_{\mathrm{p}, i}^{n}-\alpha_{\mathrm{p}, i-1}^{n} u_{\mathrm{p}, i-1}^{n}}{\Delta x} \Delta t \\
u_{\mathrm{p}, i}^{n+1}= & u_{\mathrm{p}, i}^{n}+\frac{\frac{1}{2}\left(\alpha_{\mathrm{p}, i}^{n}+\alpha_{\mathrm{p}, i-1}^{n}\right) \rho_{\mathrm{f}}\left(\frac{\alpha_{\mathrm{p}, i}^{n}\left(u_{\mathrm{p}, i}^{n}\right)^{2}-\alpha_{\mathrm{p}, i-1}^{n}\left(u_{\mathrm{p}, i-1}^{n}\right)^{2}}{\Delta x}+\frac{\alpha_{\mathrm{f}, i}^{n}\left(u_{\mathrm{f}, i}^{n}\right)^{2}-\alpha_{\mathrm{f}, i-1}^{n}\left(u_{\mathrm{f}, i-1}^{n}\right)^{2}}{\Delta x}\right)}{\alpha_{\mathrm{p}, i}^{n}\left(\alpha_{\mathrm{f}, i}^{n} \rho_{\mathrm{p}}+\alpha_{\mathrm{p}, i}^{n} \rho_{\mathrm{f}}\right)} \Delta t \\
& +\frac{\alpha_{\mathrm{p}, i}^{n} \rho_{\mathrm{f}} \frac{U^{n+1}-U^{n}}{\Delta t}+\left(\rho_{\mathrm{f}}-\rho_{\mathrm{p}}\right) \alpha_{\mathrm{p}, i}^{n} \alpha_{\mathrm{f}, i}^{n} g+F_{\mathrm{p}, i}^{n}}{\alpha_{\mathrm{p}, i}^{n}\left(\alpha_{\mathrm{f}, i}^{n} \rho_{\mathrm{p}}+\alpha_{\mathrm{p}, i}^{n} \rho_{\mathrm{f}}\right)} \Delta t+\frac{1}{2} \frac{\left(u_{\mathrm{p}, i}^{n}\right)^{2}-\left(u_{\mathrm{p}, i-1}^{n}\right)^{2}}{\Delta x} \Delta t,
\end{aligned}
$$

where $i=1,2 \mathrm{~L} \ldots I^{n+1}$ if $I^{n+1} \leq I^{n}$ or $i=1,2 \mathrm{~L} \ldots\left(I^{n+1}-1\right)$ if $I^{n+1}=I^{n}+1$. If $I^{n+1}=I^{n}+1$, at the node $i=I^{n+1}$, the following difference scheme is used,

$$
\begin{aligned}
& \alpha_{\mathrm{p}, I^{n+1}}^{n+1}=\frac{\delta^{n+1}}{\delta^{n+1}+\Delta x} \alpha_{\mathrm{p}, I^{n+1}-1}^{n+1}+\frac{\Delta x}{\delta^{n+1}+\Delta x} \alpha_{\mathrm{p}, s}^{n+1}, \\
& u_{\mathrm{p}, I^{n+1}}^{n+1}=\frac{\delta^{n+1}}{\delta^{n+1}+\Delta x} u_{\mathrm{p}, I^{n+1}-1}^{n+1}+\frac{\Delta x}{\delta^{n+1}+\Delta x} u_{\mathrm{p}, s}^{n+1},
\end{aligned}
$$


where $I^{n+1}$ is the integral grids number at time $n+1$ and defined as,

$$
I^{n+1}=\operatorname{int}\left(\frac{h^{n+1}}{\Delta x}\right)
$$

$h^{n+1}$ is the bed height and it is determined using Eq. (12),

$$
h^{n+1}=h^{n}+u_{\mathrm{p}, s}^{n} \Delta t,
$$

$u_{\mathrm{p}, s}^{n}$ is the particle velocity at the bed surface.

$\delta^{n+1}$ is the length of the fractional grid near the bed surface and defined as

$$
\delta^{n+1}=h^{n+1}-I^{n+1} \Delta x .
$$

Eq. (1) can be written as

$$
\frac{d_{p} \alpha_{\mathrm{p}}}{\mathrm{d} t}=-\alpha_{\mathrm{p}} \frac{\partial u_{\mathrm{p}}}{\partial x},
$$

where $d_{\mathrm{p}} / \mathrm{d} t=(\partial / \partial t)+u_{\mathrm{p}}(\mathrm{\partial} / \partial x)$.

Eq. (9) can be written as follows using Eqs. (1) and (8)

$$
\frac{d_{\mathrm{p}} u_{\mathrm{p}}}{\mathrm{d} t}=\frac{\alpha_{\mathrm{p}} \rho_{\mathrm{f}}\left(\frac{\partial \alpha_{\mathrm{p}} u_{\mathrm{p}}^{2}}{\partial x}+\frac{\partial \alpha_{\mathrm{f}} u_{\mathrm{f}}^{2}}{\partial x}\right)+\alpha_{\mathrm{p}} \rho_{\mathrm{f}} \frac{\partial U}{\partial t}+\left(\rho_{\mathrm{f}}-\rho_{\mathrm{p}}\right) \alpha_{\mathrm{f}} \alpha_{\mathrm{p}} g+F_{\mathrm{p}}}{\alpha_{\mathrm{p}}\left(\alpha_{\mathrm{f}} \rho_{\mathrm{p}}+\alpha_{\mathrm{p}} \rho_{\mathrm{f}}\right)} .
$$

The difference scheme of Eqs. (a-7) and (a-8) for the node at the bed surface is,

$$
\begin{aligned}
\alpha_{\mathrm{p}, s}^{n+1}= & \alpha_{\mathrm{p}, s}^{n}- \\
u_{\mathrm{p}, s}^{n+1}= & u_{\mathrm{p}, s}^{n}+\frac{\left.\frac{1}{2}\left(\alpha_{\mathrm{p}, s}^{n}+\alpha_{\mathrm{p}, I}^{n}\right) \frac{u_{\mathrm{p}, s}^{n}-u_{\mathrm{p}, I}^{n}}{\delta^{n}} \Delta t \alpha_{\mathrm{p}, I}^{n}\right) \rho_{\mathrm{f}}\left(\frac{\alpha_{\mathrm{p}, s}^{n}\left(u_{\mathrm{p}, s}^{n}\right)^{2}-\alpha_{\mathrm{p}, I}^{n}\left(u_{\mathrm{p}, I}^{n}\right)^{2}}{\delta^{n}}+\frac{\alpha_{\mathrm{f}, s}^{n}\left(u_{\mathrm{f}, s}^{n}\right)^{2}-\alpha_{\mathrm{f}, I}^{n}\left(u_{\mathrm{f}, I}^{n}\right)^{2}}{\delta^{n}}\right)}{\alpha_{\mathrm{p}, s}^{n}\left(\alpha_{\mathrm{f}, s}^{n} \rho_{\mathrm{p}}+\alpha_{\mathrm{p}, s}^{n} \rho_{\mathrm{f}}\right)} \Delta t \\
& +\frac{\alpha_{\mathrm{p}, s}^{n} \rho_{\mathrm{f}} \frac{U^{n+1}-U^{n}}{\Delta t}+\left(\rho_{\mathrm{f}}-\rho_{\mathrm{p}}\right) \alpha_{\mathrm{p}, s}^{n} \alpha_{\mathrm{f}, s}^{n} g+F_{\mathrm{p}, s}^{n}}{\alpha_{\mathrm{p}, s}^{n}\left(\alpha_{\mathrm{f}, s}^{n} \rho_{\mathrm{p}}+\alpha_{\mathrm{p}, s}^{n} \rho_{\mathrm{f}}\right)} \Delta t .
\end{aligned}
$$

\section{References}

[1] H.W. Wong, M.H.I. Baird, Chem. Eng. J. 2 (1971) 104.

[2] M. Kobayashi, D. Ramaswami, W.T. Brazelton, Chem. Eng. Prog., Symp. Ser. 66 (105) (1970) 58.

[3] S.C. Bhattacharya, D. Harrison, Inst. Chem Eng. 54 (1976) 281.

[4] M.H.I. Baird, Br. Chem. Eng. 11 (1) (1966) 20.

[5] Y. Nie, Doctoral thesis (in Chinese), Institute of Mechanics, Chinese Academy of Sciences, 1998.

[6] Y. Nie, D. Liu, Powder Technol. 99 (1998) 132.

[7] J.D. Murry, J. Fluid Mech. 21 (1965) 465.
[8] S.K. Garg, J.W. Pritchrtt, J. Appl. Phys. 46 (1975) 4493.

[9] G.M. Homsy et al., Int. J. Multiphase Flow 6 (1980) 305.

[10] Y. Tsuji, T. Tanaka, Powder Technol. 95 (1998) 254.

[11] Y.P. Tsuo, D. Gidaspow, AIChE J. 36 (6) (1990) 885.

[12] D. Gera et al., Powder Technol. 98 (1998) 38.

[13] R.D. Felice, Chem. Eng. Sci. 55 (18) (2000) 3899.

[14] D. Liu, Fluid Dynamics of Two-Phase Systems, Higher Education Press, Beijing, 1993 (in Chinese).

[15] S.C. Tsinontides, R. Jackson, J. Fluid Mech. 255 (1993) 237.

[16] P.L. Slis et al., Appl. Sci. Res., Sect. A 8 (1959) 209.

[17] J.F. Richardson, W.N. Zaki, Trans. Inst. Chem. Eng. 32 (1954) 35. 